\title{
Block Markov superposition transmission with pulse position modulation over free-space optical links
}

\author{
Jinshun Zhu ${ }^{1}$, Shancheng Zhao ${ }^{2}$, Xiao $\mathrm{Ma}^{3,4 *}$ \\ 1. School of Electronics and Information Technology, Sun Yat-sen University, Guangzhou 510006, China \\ 2. College of Information Science and Technology, Jinan University, Guangzhou 510632, China \\ 3. School of Data and Computer Science, Sun Yat-sen University, Guangzhou 510006, China \\ 4. Guangdong Key Laboratory of Information Security Technology, Sun Yat-sen University, Guangzhou 510006, China \\ *Corresponding author, Email: maxiao@mail.sysu.edu.cn
}

\begin{abstract}
In FSO (Free-Space Optical) communications, performance of the communication systems is severely degraded by atmospheric turbulence. PPM (Pulse Position Modulation) is widely used in FSO communication systems owing to its high power efficiency. In this paper, we present a combination of the BMST (Block Markov Superposition Transmission) technique and the PPM scheme to improve the reliability of the transmission over FSO links. Based on analyzing an equivalent system, a lower bound on the bit-error-rate of the proposed scheme is presented. Extensive simulations are performed which show that the BMST-PPM system performs well under a wide range of turbulence conditions and improves the performance of the basic code. Simulation results also show that, the performance of the system with the sliding-window detection/decoding algorithm matches well with the lower bound in the low-error-rate region.
\end{abstract}

Keywords: BMST, FSO, gamma-gamma channel model, iterative detection/decoding algorithm, PPM

Citation: J. S. Zhu, S. C. Zhao, X. Ma. Block Markov superposition transmission with pulse position modulation over free-space optical links [J]. Journal of communications and information networks, 2017, 2(4): $142-150$.

\section{Introduction}

Recently, FSO communications have attracted considerable attention owing to their advantages: large bandwidth, license free spectrum, inherent security, high data rate, and fast deployment ${ }^{[1]}$. With these virtues, FSO communications find applications in satellite communications, last mile access, backhaul for RF (Radio Frequency) systems, and so on ${ }^{[2,3]}$. FSO communication systems are well established over terrestrial links, ground-to-satellite/satellite-to- ground links, or inter-satellite/deep-space links ${ }^{[4-8]}$.

In FSO communications, performance of the communication systems is affected by the misalignmentinduced pointing errors over point-to-point lineof-sight links. FSO communications over terrestrial links and ground-to-satellite/satellite-to-ground links are also affected by weather conditions. Even under clear sky conditions, the performance of the FSO communication systems is severely degraded by atmospheric turbulence ${ }^{[9]}$. Atmospheric turbulence is a natural phenomenon caused by inhomogeneities

Manuscript received Sep. 23, 2017; accepted Nov. 10, 2017

This work is partially supported by the Basic Research Project of Guangdong Provincial Natural Science Foundation (No. 2016A030308008), the National Natural Science Foundation of China (Nos. 91438101 and 61501206), and the National Basic Research Program of China ("973" Program) (No. 2012CB316100). 
in the temperature and pressure of the atmosphere, which leads to the refractive index variations along the transmission paths. The atmospheric turbulence produces random fluctuations in both amplitude and phase of the received signal, i.e., channel fading. In this paper, we assume that the transceivers are perfectly aligned. We also assume that the communication system is under clear sky conditions, and focus on mitigating the channel fading caused by atmospheric turbulence.

To improve the reliability over atmospheric turbulence channels, FSO communications can employ mitigation techniques such as channel coding techniques $^{[10-16]}$, diversity techniques ${ }^{[17,18]}$, adaptive transmission $^{[19,20]}$, relay-assisted transmission ${ }^{[21,22]}$, and hybrid RF/FSO ${ }^{[23,24]}$. Owing to their good performance and easy implementation, channel coding techniques, including CC (Convolutional Codes), turbo codes and LDPC (Low-Density Parity-Check) codes, are commonly used in the FSO communication systems ${ }^{[10-16]}$. In FSO communications, IM/DD (Intensity Modulation with Direct Detection) is used in the most current optical communication systems. OOK (On-Off Keying) modulation and PPM (Pulse Position Modulation) are two most commonly used intensity modulation techniques. Compared with OOK modulation, PPM is more power efficient and provides a high PAPR (Peak-to-Average Power Ratio). Hence, it is widely used for long-distance or deep-space communications ${ }^{[25,26]}$.

BMST (Block Markov Superposition Transmission) is a recently proposed coding scheme ${ }^{[27]}$. In the BMST scheme, binary sequences are encoded with a basic code and then transmitted multiple times (in their interleaved versions) to improve the transmission reliability. Meanwhile, the interleaved sequences are superimposed in a block Markov manner to maintain the transmission efficiency. Any short code (linear or nonlinear) with a fast encoding algorithm and an efficient SISO (Soft-In SoftOut) decoding algorithm can be chosen as the basic code ${ }^{[27]}$. BMST also has a simple but tight performance lower bound. Furthermore, BMST is effective in constructing capacity-approaching cod- ing schemes for AWGN (Additive White Gaussian Noise) channels ${ }^{[27]}$. However, its combination with PPM over FSO links has never been investigated in the literature.

In this paper, we propose to employ BMST codes to improve the reliability of the FSO links with PPM. We present a lower bound on the BER (Bit-ErrorRate) of the proposed transmission scheme by analyzing an equivalent system. To show the advantages of the proposed scheme, we perform extensive simulations. These simulation results show that: First, the BMST-PPM system performs well under a wide range of turbulence conditions. Second, the performance of the system with the sliding-window detection/decoding algorithm matches well with the lower bound in the low-error-rate region.

\section{Channel model}

In studies on FSO communications, statistical models are commonly used to characterize the channel fading caused by atmospheric turbulence ${ }^{[9,17,28,29]}$. The log-normal model is widely employed under weak turbulence conditions ${ }^{[9,28]}$, while the K-distribution model can suitably describe the irradiance fluctuation under strong turbulence conditions ${ }^{[9,17]}$. The gamma-gamma model fits a wide range of turbulence regimes (from weak to strong) well ${ }^{[9,29]}$. In this paper, the gamma-gamma model is adopted to describe the fading statistics of the channel. In the gamma-gamma model, the received intensity $I$ is defined as the product of two statistically independent random variables $I_{x}$ and $I_{y}$, which arise from large-scale and small-scale turbulent eddies, respectively. The PDF (Probability Density Function) of the normalized intensity $I=I_{x} I_{y}$ is given by ${ }^{[9,29]}$

$$
\begin{array}{r}
p(I)=\frac{2(\alpha \beta)^{(\alpha+\beta) / 2}}{\Gamma(\alpha) \Gamma(\beta)} I^{(\alpha+\beta) / 2-1} K_{\alpha-\beta}(2 \sqrt{\alpha \beta I}), \\
I>0, \quad(1)
\end{array}
$$

where $\alpha$ and $\beta$ are the effective numbers of largescale and small-scale eddies, respectively, $K_{a}(\cdot)$ is the modified Bessel function of the second kind of 
order $a$, and $\Gamma(\cdot)$ is the gamma function. Assuming plane wave propagation, the two parameters $\alpha$ and $\beta$ are directly related to atmospheric conditions according to ${ }^{[9,29]}$

$$
\begin{aligned}
& \alpha=\left[\exp \left(\frac{0.49 \sigma_{R}^{2}}{\left(1+1.11 \sigma_{R}^{12 / 5}\right)^{7 / 6}}\right)-1\right]^{-1}, \\
& \beta=\left[\exp \left(\frac{0.51 \sigma_{R}^{2}}{\left(1+0.69 \sigma_{R}^{12 / 5}\right)^{5 / 6}}\right)-1\right]^{-1},
\end{aligned}
$$

where $\sigma_{R}^{2}=1.23 C_{n}^{2} k^{7 / 6} L_{p}^{11 / 6}$ is the Rytov variance. Here, $C_{n}^{2}$ is the refractive index structure parameter, $k=2 \pi / \lambda$ is the optical wave number, $\lambda$ is the wavelength, and $L_{p}$ is the propagation distance. Weak fluctuations are associated with $\sigma_{R}^{2}<1$, the moderate with $\sigma_{R}^{2} \approx 1$, and the strong with $\sigma_{R}^{2}>1.0^{[9]}$.

In this paper, we consider PPM as the modulation scheme of the system. Let $\underline{x}=\left(x_{0}, x_{1}, \cdots, x_{Q-1}\right)$ be a modulated PPM symbol, where $x_{j}$ is the subsymbol corresponding to the $j$-th slot of the $Q$ slots of a PPM symbol. Here, we refer to $\underline{x}$ as a slotword. When the $j$-th sub-symbol of a slot-word $\underline{x}$ is transmitted, the received electrical signal after the optical/electrical conversion is

$$
y_{j}=\eta I x_{j}+z_{j},
$$

where $\eta$ is the optical/electrical conversion efficiency, $I$ is a sample from a gamma-gamma distribution, and $z_{j}$ is the sum of thermal, dark, and shot noise. We assume that the receiver is thermal-noise limited and hence $z_{j}$ is a zero-mean white Gaussian noise with two-sided power spectral density $N_{0} / 2$. Without loss of generality, we set $\eta=1$. Following Refs. [11,12], we assume that the CSI (Channel State Information) is perfectly known at the receiver and the channel fades corresponding to the PPM symbols are independent and identically distributed.

\section{$3 \quad$ BMST with PPM}

\subsection{The transmitter}

Let $\boldsymbol{u}^{(0)}, \boldsymbol{u}^{(1)}, \cdots, \boldsymbol{u}^{(L-1)}$ be $L$ sub-blocks of data to be transmitted, where $0 \leqslant t \leqslant L-1$ and $\boldsymbol{u}^{(t)} \in \mathbb{F}_{2}^{k}$.
Let $\mathcal{C}[n, k]$ be a binary code with length $n$ and dimension $k$, which is referred to as a basic code. Let $\mathcal{X}$ be a signal constellation of size $2^{b}$ and $\varphi: \mathbb{F}_{2}^{b} \rightarrow \mathcal{X}$ be the one-to-one mapping corresponding to the PPM scheme. The encoding process with memory $m$ is described as follows. We can take Fig. 1 for reference.

Algorithm 1: The encoding/modulation algorithm of the BMST-PPM system

- Initialization: For $t<0$, set $\boldsymbol{v}^{(t)}=\mathbf{0} \in \mathbb{F}_{2}^{n}$.

- Loop: For $t=0,1, \cdots, L-1$,

1. Encoding: Encode $\boldsymbol{u}^{(t)}$ into $\boldsymbol{v}^{(t)} \in \mathbb{F}_{2}^{n}$ by the encoding algorithm of the basic code $\mathcal{C}[n, k]$;

2. Interleaving: For $0 \leqslant i \leqslant m$, interleave $\boldsymbol{v}^{(t-i)}$ into $\boldsymbol{w}^{(i)}$ by the $i$-th interleaver $\boldsymbol{\Pi}_{i}$;

3. Superposition: Compute $\boldsymbol{c}^{(t)}=\sum_{0 \leqslant i \leqslant m} \boldsymbol{w}^{(i)}$;

4. Mapping: Rewrite the $t$-th sub-codeword as $c^{(t)}=$ $\left(\underline{c}_{0}^{(t)}, \cdots, \underline{c}_{N-1}^{(t)}\right)$, where $\underline{c}_{j}^{(t)} \in \mathbb{F}_{2}^{b}$ for $0 \leqslant j \leqslant N-1$. Then map $\boldsymbol{c}^{(t)}$ into a transmission signal sequence $\boldsymbol{x}^{(t)}=\left(x_{0}^{(t)}, x_{1}^{(t)}, \cdots, x_{N-1}^{(t)}\right)$ according to the PPM scheme, where $x_{j}^{(t)}=\varphi\left(\underline{c}_{j}^{(t)}\right)$ for $0 \leqslant j \leqslant N-1$.

- Termination: For $t=L, L+1, \cdots, L+m-1$, set $\boldsymbol{u}^{(t)}=\mathbf{0} \in \mathbb{F}_{2}^{k}$ and compute the transmission signal sequence $\boldsymbol{x}^{(t)}$ following the steps in Loop.

\subsection{The receiver}

The detection/decoding algorithm at the receiver in the BMST-PPM system can be described as an iterative message processing/passing algorithm over the associated normal graph. In the normal graph, edges represent variables and vertices (nodes) represent constraints. All edges connected to a node must satisfy the specific constraint of the node. The normal graph of a BMST-PPM system with $L=4$ and $m=2$ is shown in Fig. 2. The normal graph of the BMST-PPM system can be divided into layers. Each layer typically consists of a node of type C, a node of type $=, m$ nodes of type $\Pi$, and a node of type + that is connected to a node of type $\varphi$. The four types of nodes in each layer are the same as those in the original BMST system ${ }^{[27]}$, while the node $\varphi$ represents the constraint introduced by the PPM. In this paper, a message for a discrete random variable is defined as its probability mass function. Upon receiving $\boldsymbol{y}^{(t+d)}$, the BMST-PPM system 


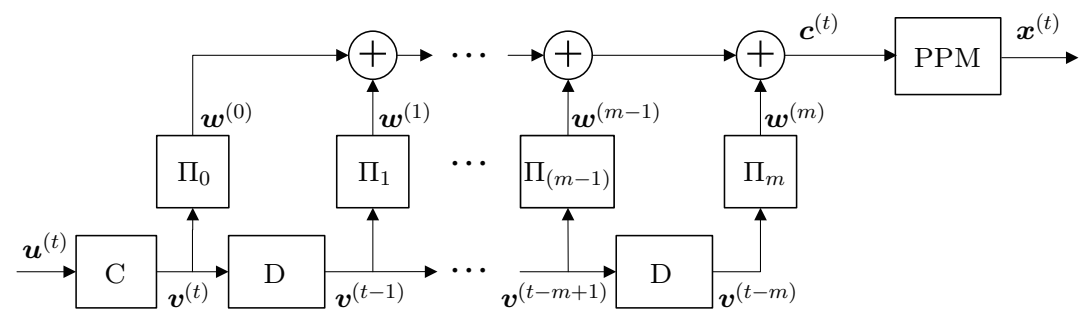

Figure 1 Block diagram of the BMST-PPM system of memory $m$

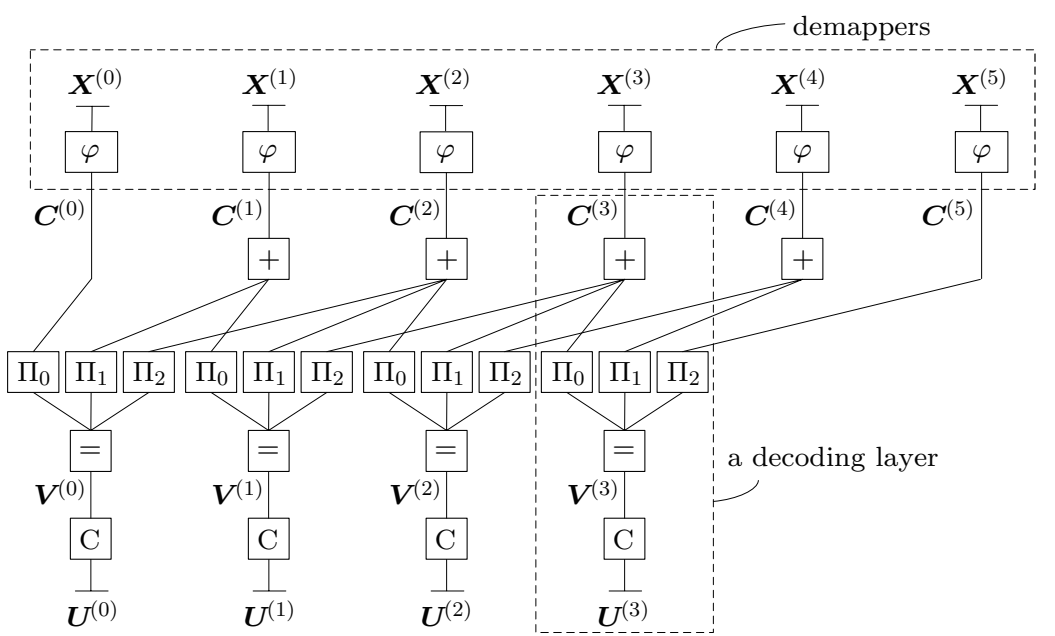

Figure 2 Normal graph for a BMST-PPM system $(L=4, m=2)$

performs a sliding-window detection/decoding algorithm with a decoding delay $d$ to recover $\boldsymbol{u}^{(t)}$. The following detection/decoding algorithm is similar to that described in Ref. [27].

Algorithm 2: The iterative sliding-window detection/decoding algorithm for the BMST-PPM system

- Global initialization: Assume that $\boldsymbol{y}^{(t)}(0 \leqslant t \leqslant d-1)$ has been received. For $0 \leqslant t \leqslant d-1,0 \leqslant j \leqslant N-1$, and $0 \leqslant \ell \leqslant b-1$, compute $P\left(C_{j, \ell}^{(t)}=a\right)$ at the node $\varphi$,

$$
P\left(C_{j, \ell}^{(t)}=a\right) \propto \sum_{c_{j, \ell}^{(t)}=a, \underline{c}_{j}^{(t)} \in \in_{2}^{b}} P\left(\underline{y}_{n}^{(t)} \mid \varphi\left(\underline{c}_{j}^{(t)}\right)\right),
$$

where $a \in \mathbb{F}_{2}$. All messages over the other edges within and connecting to the $t$-th layer $(0 \leqslant t \leqslant d-1)$ are initialized as uniformly distributed variables. Set a maximum iteration number $J_{\max }>0$. Set a threshold $\epsilon>0$ and initialize the entropy rate $h_{0}\left(\boldsymbol{Y}^{(t)}\right)=0$, where $\boldsymbol{Y}^{(t)}$ is the random vector corresponding to $\boldsymbol{y}^{(t)}$.

- Sliding-window decoding: For $t=0,1, \cdots, L-1$,

1. Local initialization: If $t+d \leqslant L+m-1$, compute $P\left(C_{j, \ell}^{(t)}=a\right)$ at the node $\varphi$ by Eq. (5). All messages over the other edges within and connecting to the $t$-th layer are initialized as uniformly distributed variables. Initialize the entropy rate $h_{0}\left(\boldsymbol{Y}^{(t)}\right)=0$.
2. Iteration: For $J=1,2, \cdots, J_{\max }$,

(a) Forward recursion: For $i=0,1, \cdots, \min (d$, $L+m-1-t)$, the $(t+i)$-th layer performs a message processing/passing algorithm schedule as $+\rightarrow=\rightarrow \mathrm{C} \rightarrow=\rightarrow \Pi \rightarrow+$

(b) Backward recursion: For $i=\min (d, L+m-1-t)$, $\cdots, 1,0$, the $(t+i)$-th layer performs a message processing/passing algorithm schedule as $+\rightarrow \rightarrow \rightarrow \mathrm{C} \rightarrow=\rightarrow \Pi \rightarrow+$.

(c) Hard decision: Make hard decision to obtain the estimation $\hat{\boldsymbol{u}}^{(t)}$ corresponding to $\boldsymbol{u}^{(t)}$. Estimate the entropy rate $h_{J}\left(\boldsymbol{Y}^{(t)}\right)$ of $\boldsymbol{Y}^{(t)}[27]$. Output $\hat{\boldsymbol{u}}^{(t)}$ and exit the iteration, if $\left|h_{J}\left(\boldsymbol{Y}^{(t)}\right)-h_{J-1}\left(\boldsymbol{Y}^{(t)}\right)\right| \leqslant \epsilon$.

\section{Genie-aided lower bound}

In the BMST-PPM system, each single data block is involved in $m+1$ consecutive transmissions by Markov superposition, resulting in interference among adjacent data blocks and possible error propagation in the process of data recovery. Simi- 
lar to the analysis in Ref. [27], the BER performance of the BMST-PPM system can be lower bounded by a genie-aided decoder, in which all but one of the data blocks are known. That is, with knowledge of CSI, the genie-aided decoder computes $\operatorname{Pr}\left\{U_{j}^{(t)}=a \mid \boldsymbol{u}^{\prime}, \boldsymbol{y}^{(0)}, \cdots, \boldsymbol{y}^{(L+m-1)}\right\}$ for all $a, t$ and $j$ with the data $\boldsymbol{u}^{\prime}=\left(\boldsymbol{u}^{(0)}, \cdots\right.$, $\left.\boldsymbol{u}^{(t-1)}, \boldsymbol{u}^{(t+1)}, \cdots, \boldsymbol{u}^{(L-1)}\right)$ available. As shown in Fig. 3, the encoding and mapping process for the equivalent system with a basic code $\mathcal{C}[n, k]$ is described as follows:

- Encode $\boldsymbol{u}^{(t)} \in \mathbb{F}_{2}^{k}$ into a codeword $\boldsymbol{v}^{(t)} \in \mathbb{F}_{2}^{n}$ by the encoding algorithm of the basic code;

- Interleave $\boldsymbol{v}^{(t)}$ into $m+1$ versions $\boldsymbol{w}^{(0)}, \cdots, \boldsymbol{w}^{(m)}$ by $m+1$ interleavers in parallel;

- Map $\boldsymbol{w}^{(i)}$ into a signal sequence $\boldsymbol{s}^{(i)}$ of length $N$ according to the PPM for $0 \leqslant i \leqslant m$.

- Output $\boldsymbol{x}^{(t)}=\left(\boldsymbol{s}^{(0)}, \cdots, \boldsymbol{s}^{(m)}\right)$ of length $N(m+$ 1) as the block of transmission signal sequence.

The detection/decoding algorithm for the equivalent system can be described as a message processing/passing algorithm over the associated normal graph, as shown in Fig. 4. The nodes of type $\varphi, \Pi, \quad=$ and $\mathrm{C}$ are the same with those in the normal graph of the BMST-PPM system. Here the node $\mathrm{P} / \mathrm{S}$ represents the parallel-to-serial conversion. The detection/decoding algorithm for the equivalent system is described as follows:

- Initialization: Compute the messages associated with $\boldsymbol{w}^{(t)}$ at the node $\varphi$ by Eq. (5). All messages over the edges connected to the nodes $\Pi,=$ and C are initialized as uniformly distributed variables.

- Message processing/passing: Perform a message processing/passing algorithm scheduled as $\varphi \rightarrow$ $\Pi \rightarrow=\rightarrow \mathrm{C}$.

- Hard decision: Make a hard decision to obtain the estimation $\hat{\boldsymbol{u}}^{(t)}$ corresponding to $\boldsymbol{u}^{(t)}$.

In this paper, the mean SNR per symbol is defined as $\bar{\gamma}_{s}=E\left[I^{2}\right] \gamma_{s}=(1+1 / \alpha)(1+1 / \beta) E_{s} / N_{0}$, and the mean SNR per information bit is given by $\bar{\gamma}_{b}=E_{b} / N_{0} \triangleq \bar{\gamma}_{s} /(b R)$, where $R$ is the code rate. Let $p_{b}=f_{\text {EquSys }}\left(\bar{\gamma}_{b}\right)$ and $p_{b}=f_{\mathrm{BMST}-\mathrm{PPM}}\left(\bar{\gamma}_{b}\right)$ be the BER performance functions of the equivalent sys- tem and the BMST-PPM system, respectively, where $p_{b}$ is the BER and $\bar{\gamma}_{b}$ is in $\mathrm{dB}$. With a similar argument given in Ref. [27], and the rate loss taken into account, the BER performance of the BMST-PPM system can be lower bounded as

$$
\begin{aligned}
f_{\mathrm{BMST}-\mathrm{PPM}}\left(\bar{\gamma}_{b}\right) \geqslant & f_{\mathrm{EquSys}}\left(\bar{\gamma}_{b}+10 \log (m+1)\right. \\
& -10 \log (1+m / L)) .
\end{aligned}
$$

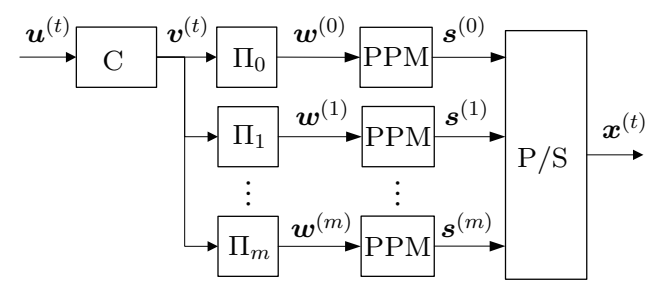

Figure 3 Equivalent system corresponding to the genieaided decoder for the BMST-PPM system of memory $m$

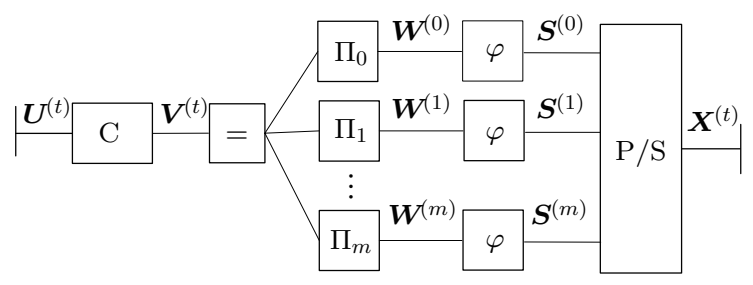

Figure 4 Normal graph for the equivalent system with memory $m$

\section{Numerical results}

In this section, we present several examples to investigate the performance of the BMST-PPM systems under weak, moderate, and strong turbulence conditions. We set $\sigma_{R}^{2}$ to $0.04,1.0$ and 9.0 for the cases of weak, moderate and strong turbulence conditions, respectively. The interleavers used in the BMSTPPM system are randomly generated but fixed. We choose a terminated 4-state $(2,1,2)$ CC (Convolutional Code) as the basic code, which is defined by the polynomial generator matrix $G(D)=$ $\left[1+D^{2}, 1+D+D^{2}\right]$. We adopt the BCJR (BahlCocke-Jelinek-Raviv) algorithm as the soft-in softout decoding algorithm of the basic code. The iterative sliding-window detection/decoding algorithm performs with a maximum iteration number of 18 , and the entropy stopping criterion is employed with 
a preselected threshold $\epsilon=10^{-5}$. Unless stated otherwise, in our simulations, the termination length is $L=1000$ and the information length is $k=5000$. The decoding delay is chosen as $d=3 \mathrm{~m}$.

Example 1: In this example, we consider the BMST system with 4PPM under weak turbulence conditions. For weak turbulence conditions, the Rytov variance is set to $\sigma_{R}^{2}=0.04$, resulting in $\alpha=51.9$ and $\beta=49.1$. The BER performance of the BMSTPPM system is shown in Fig. 5. From Fig. 5, we have the following observations:

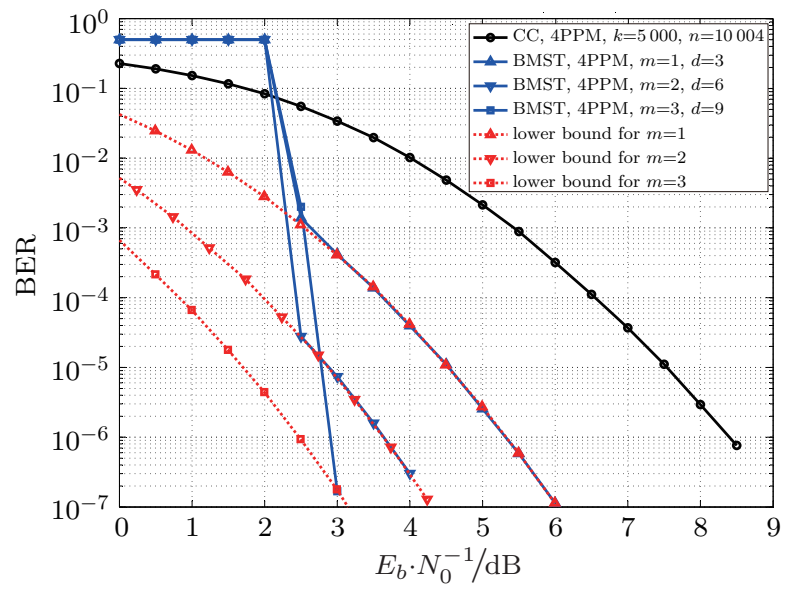

Figure 5 Performance of the BMST system with 4PPM under weak turbulence conditions

1. In the BMST-PPM system, interference among adjacent data blocks is introduced by Markov superposition, which leads to possible error propagation in the process of data recovery. For this reason, the iterative sliding-window detection/decoding algorithm cannot work well in the low SNR region. It is obvious that, a larger memory $m$ results in more severe error propagation.

2. Compared with the case in the low SNR region, the BMST-PPM system performs well and approaches its lower bounds in the high SNR region, where the effect of error propagation is effectively mitigated adopting the iterative sliding-window detection/decoding algorithm. Hence, there is a waterfall region between the low SNR region and the high SNR region in each performance curve of the BMST-PPM system.
3. The BMST-PPM system improves the BER performance of the basic code in the high SNR region. Compared with the basic code, the BMST system with memory $m=1$ and 2 gains about $3.0 \mathrm{~dB}$ and $4.7 \mathrm{~dB}$ at the BER of $10^{-5}$, respectively.

4. In the high SNR region, the performance of the BMST system improves with the increase of memory. Example 2: In this example, we perform performance comparisons between the BMST systems with different PPM schemes under weak turbulence conditions, when memory $m=1$ and 2. From Figs. 6 and 7 , we observe that, the BER performance of the BMST-PPM system in the error floor region im-

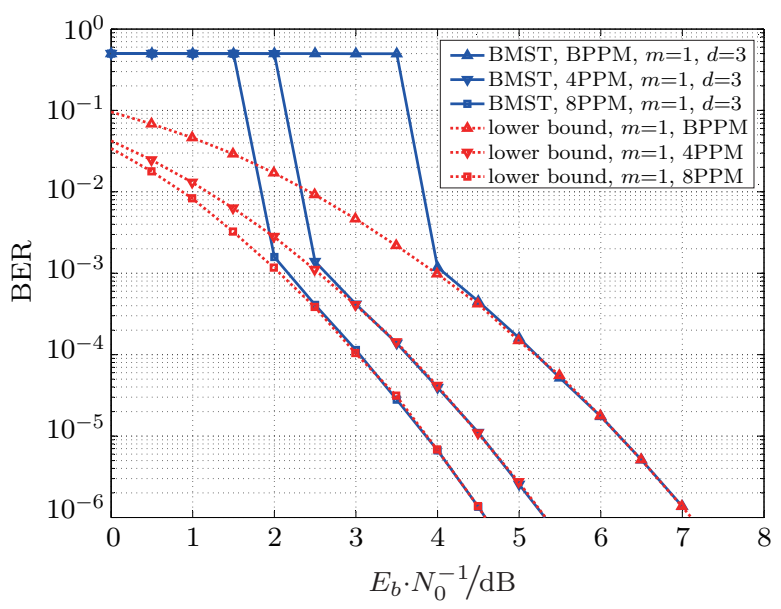

Figure 6 Performance comparisons of the BMST systems with BPPM, 4PPM and 8PPM under weak turbulence conditions $(m=1)$

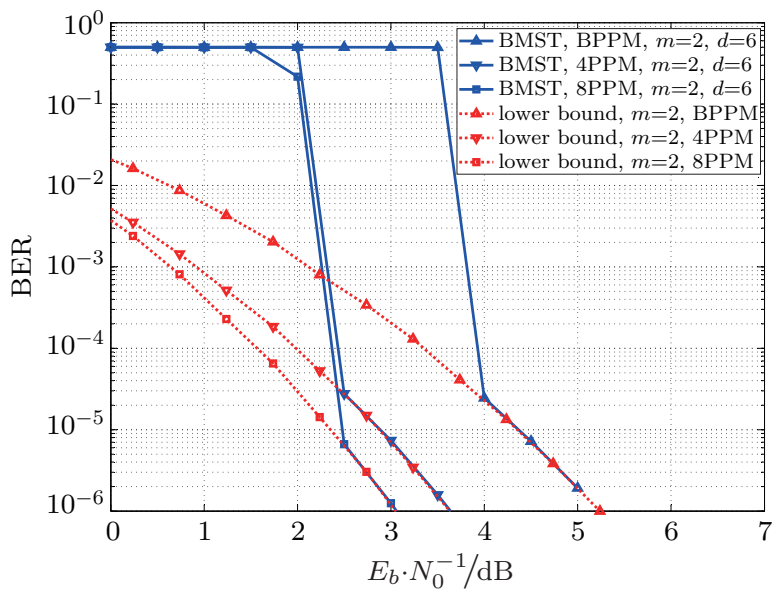

Figure 7 Performance comparisons of the BMST systems with BPPM, 4PPM and 8PPM under weak turbulence conditions $(m=2)$ 
proves with the increase of the order of the PPM scheme. This means that the BMST system with higher-order PPM is more power efficient. We also observe that the BER performance of the BMSTPPM system in the waterfall region also improves with the increase of the order of the PPM scheme.

Example 3: In this example, we consider the BMST system with 4PPM under moderate and strong turbulence conditions. For moderate turbulence conditions, the Rytov variance is set to $\sigma_{R}^{2}=1.0$, resulting in $\alpha=4.39$ and $\beta=2.56$. For strong turbulence conditions, the Rytov variance is set to $\sigma_{R}^{2}=9.0$, resulting in $\alpha=5.49$ and $\beta=1.12$. The BER performance of the BMST-PPM system is shown in Figs. 8 and 9. From Figs. 8 and 9, we observe that the BMST

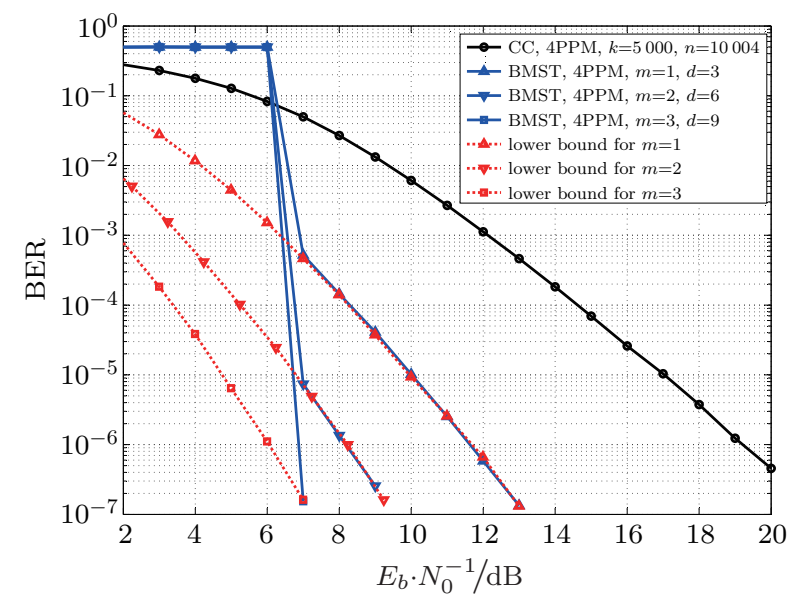

Figure 8 Performance of the BMST system with 4PPM under moderate turbulence conditions

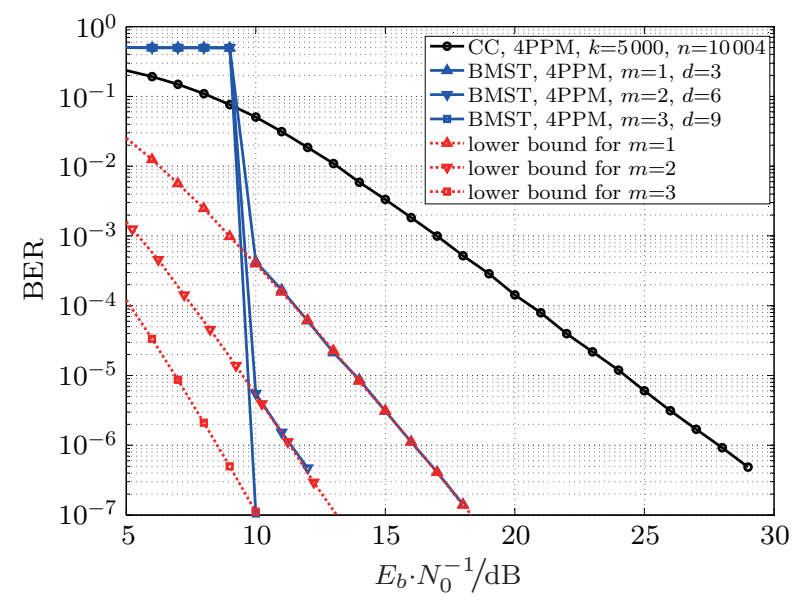

Figure 9 Performance of the BMST system with 4PPM under strong turbulence conditions system with PPM also performs well under moderate and strong turbulence conditions. In the high SNR region, the performance curves match well with their corresponding lower bounds. When the effect of the atmospheric turbulence becomes more severe, the BMST-PPM system gains more improvement compared with the basic code, and gains more improvement with the increase of the memory $m$.

Remarks: For the basic code $\mathcal{C}[n, k]$ (e.g., a convolutional code) which can be represented by a trellis with $\gamma$ branches per coded bit, the decoding complexity with the BCJR algorithm is $O(\gamma n)$. As pointed out in Ref. [27], for a BMST system with memory $m$, a basic code $\mathcal{C}[n, k]$ and a decoding delay $d$, the decoding complexity at each iteration is $O((2(m+2)+\gamma)(d+1) n)$. The decoding complexity also relies on the iteration number. We found that to recover one layer with a properly designed stopping criterion, 2 iterations on average (in the low BER region) are required in our simulations.

\section{Conclusion and future work}

In this paper, we propose to use block Markov superposition transmission with PPM over FSO links. Based on analyzing an equivalent system, a lower bound on the BER performance of the proposed scheme is presented. Extensive simulations have been performed to investigate the performance of BMST with PPM over FSO links. The simulation results show that the BMST-PPM system performs well under a wide range of turbulence conditions and outperforms its basic codes. The BER performance of the system with the sliding-window detection/decoding algorithm matches well with the lower bound in the low-error-rate region. In future studies, we will consider the iteration between the BMST decoder and the PPM detector, the combination of BMST and multiple-PPM, and further performance analyses.

\section{References}

[1] M. Uysal, C. Capsoni, Z. Ghassemlooy, et al. Optical wireless communications: an emerging technology $[\mathrm{M}]$. 
Berlin: Springer, 2016.

[2] M. A. Khalighi, M. Uysal. Survey on free space optical communication: a communication theory perspective [J]. IEEE communications surveys \& tutorials, 2014, 16(4): 2231-2258.

[3] H. Kaushal, G. Kaddoum. Optical communication in space: challenges and mitigation techniques [J]. IEEE communications surveys \& tutorials, 2017, 19(1): 57-96.

[4] E. Ciaramella, Y. Arimoto, G. Contestabile, et al. 1.28 terabit/s (3240 Gbit/s) WDM transmission system for free space optical communications [J]. IEEE journal on selected areas in communications, 2009, 27(9):16391645.

[5] M. Toyoda, M. Toyoshima, T. Takahashi, et al. Ground-to-ETS-VI narrow laser beam transmission [C]//Proceedings of SPIE, 1996: 71-80.

[6] K. E. Wilson. An overview of the GOLD experiment between the ETS- 6 satellite and the table mountain facility [R]. TDA Progress Report, 1996: 8-19.

[7] A. Katsuyoshi. 2 overview of the optical inter-orbit communications engineering test satellite (OICETS) project $[\mathrm{J}]$. Journal of the national institute of information and communications technology, 2012, 59: 5-12.

[8] A. Biswas, D. Boroson, B. Edwards. Mars laser communication demonstration: What it would have been [C]//Proceedings of SPIE, 2006, 6105(02): 1-12.

[9] L. C. Andrews, R. L. Phillips. Laser beam propagation through random media $[\mathrm{M}]$. Bellingham, WA: SPIE Press, 2005.

[10] J. Anguita, I. Djordjevic, M. Neifeld, et al. Shannon capacities and error-correction codes for optical atmospheric turbulent channels [J]. Journal of optical networking, 2005, 4(9): 586-601.

[11] M. Uysal, J. Li, M. Yu. Error rate performance analysis of coded free-space optical links over gamma-gamma atmospheric turbulence channels [J]. IEEE transactions on wireless communications, 2006, 5(6): 1229-1233.

[12] W. Gappmair, M. Flohberger. Error performance of coded FSO links in turbulent atmosphere modeled by gamma-gamma distributions [J]. IEEE transactions on wireless communications, 2009, 8(5): 2209-2213.

[13] F. Xu, A. Khalighi, P. Caussé, et al. Channel coding and time-diversity for optical wireless links [J]. Optics express, 2009, 17(2): 872-887.

[14] Y. Q. Han, A. H. Dang, Y. X. Ren, et al. Theoretical and experimental studies of turbo product code with time diversity in free space optical communication $[\mathrm{J}]$. Optics express, 2010, 18(26): 26978-26988.

[15] N. Cvijetic, S. G. Wilson, R. Zarubica. Performance evaluation of a novel converged architecture for digitalvideo transmission over optical wireless channels [J]. Journal of lightwave technology, 2007, 25(11): 33663373.

[16] I. B. Djordjevic, S. Denic, J. Anguita, et al. LDPC- coded MIMO optical communication over the atmospheric turbulence channel [J]. Journal of lightwave technology, 2008, 26(5): 478-487.

[17] T. A. Tsiftsis, H. G. Sandalidis, G. K. Karagiannidis, et al. Optical wireless links with spatial diversity over strong atmospheric turbulence channels [J]. IEEE transactions on wireless communications, 2009, 8(2): 951957.

[18] H. E. Nistazakis, G. S. Tombras. On the use of wavelength and time diversity in optical wireless communication systems over gamma-gamma turbulence channels [J]. Optics \& laser technology, 2012, 44(7): 2088-2094.

[19] M. Karimi, M. Uysal. Novel adaptive transmission algorithms for free-space optical links [J]. IEEE transactions on communications, 2012, 60(12): 3808-3815.

[20] I. B. Djordjevic. Adaptive modulation and coding for free-space optical channels [J]. IEEE journal of optical communications and networking, 2010, 2(5): 221-229.

[21] H. Alquwaiee, I. S. Ansari, M. S. Alouini. On the performance of free-space optical communication systems over double generalized gamma channel [J]. IEEE journal on selected areas in communications, 2015, 33(9): 1829-1840.

[22] S. M. Aghajanzadeh, M. Uysal. Performance analysis of parallel relaying in free-space optical systems [J]. IEEE transactions on communications, 2015, 63(11): 43144326.

[23] Y. Tang, M. Brandt-Pearce, S. G. Wilson. Link adaptation for throughput optimization of parallel channels with application to hybrid FSO/RF systems [J]. IEEE transactions on communications, 2012, 60(9): 27232732 .

[24] V. Jamali, D. S. Michalopoulos, M. Uysal, et al. Link allocation for multiuser systems with hybrid RF/FSO backhaul: delay-limited and delay-tolerant designs [J]. IEEE transactions on wireless communications, 2016, 15(5): 3281-3295.

[25] H. Hemmati. Deep space optical communication [Z]. New York, NY, USA: Wiley, 2006.

[26] B. Moision, J. Hamkins. Deep-space optical communications downlink budget: modulation and coding [R]. IPN Progress Report, 2003, 42(154): 1-28.

[27] X. Ma, C. L. Liang, K. C. Huang, et al. Block Markov superposition transmission: construction of big convolutional codes from short codes [J]. IEEE transactions on information theory, 2015, 61(6): 3150-3163.

[28] X. M. Zhu, J. M. Kahn. Free-space optical communication through atmospheric turbulence channels [J]. IEEE transactions on communications, 2002, 50(8): 12931300 .

[29] M. A. Al-Habash, L. C. Andrews, R. L. Phillips. Mathematical model for the irradiance probability density function of a laser beam propagating through turbulent media $[\mathrm{J}]$. Optical engineering, 2001, 40(8): 1554-1562. 


\section{About the authors}

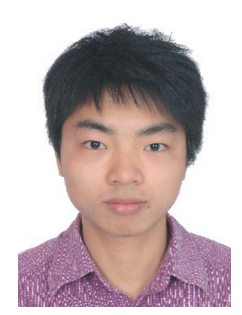

Jinshun Zhu was born in Hunan, China. He is currently a Ph.D. candidate at Sun Yat-sen University, Guangzhou, China. His current research interests include channel coding theory, coded modulation techniques, and their applications in freespace optical communications. (Email: zhujinsh@mail2.sysu.edu.cn)

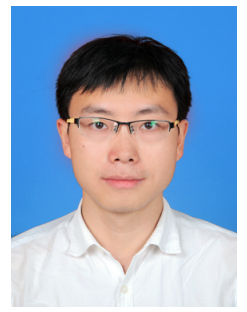

Shancheng Zhao was born in Sichuan, China. He received his bachelor's and Ph.D. degrees from Sun Yat-sen University in 2009 and 2014, respectively. He is currently an assistant professor with the College of Information Science and Technology, Jinan University, Guangzhou, China. From 2013 to 2014, he was a graduate visiting student with UCLA, CA. His current research interests include LDPC codes and their applications in storage systems and visible light communication systems. He is a co-recipient of the Best Paper Award of 2015 IEEE GlobeCom. (Email: zhaoday@mail2.sysu.edu.cn)

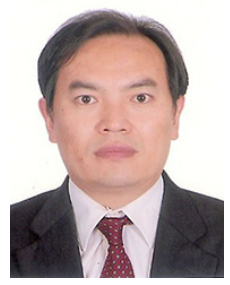

Xiao Ma [corresponding author] was born in Henan, China. He received his Ph.D. degree in communication and information systems from Xidian University, China, in 2000. From 2000 to 2002, he was a post-doctoral fellow with Harvard University, Cambridge, MA. From 2002 to 2004 , he was a research fellow with the

City University of Hong Kong. He is currently a professor with the School of Data and Computer Science, Sun Yat-sen University, Guangzhou, China. His research interests include information theory, channel coding theory and their applications in communication systems and digital recording systems. He is a co-recipient, with A. Kavcic and N. Varnica, of the 2005 IEEE Best Paper Award in Signal Processing and Coding for Data Storage. In 2006, he received the Microsoft Professorship Award from Microsoft Research Asia. (Email: maxiao@mail.sysu.edu.cn) 